\title{
A Note on Acyclic Edge Colouring of Star Graph Families
}

\author{
P. Shanasbabu, A. V. Chithra \\ Department of Mathematics, National Institute of Technology, Calicut, India \\ Email: babushanas@gmail.com
}

Received 2 July 2015; accepted 22 August 2015; published 26 August 2015

Copyright (C 2015 by authors and Scientific Research Publishing Inc.

This work is licensed under the Creative Commons Attribution International License (CC BY). http://creativecommons.org/licenses/by/4.0/

(c) ()

\section{Abstract}

A proper edge colouring $\boldsymbol{f}$ of a graph $\boldsymbol{G}$ is called acyclic if there are no bichromatic cycles in the graph. The acyclic edge chromatic number or acyclic chromatic index, denoted by $a^{\prime}(G)$, is the minimum number of colours in an acyclic edge colouring of $G$. In this paper, we discuss the acyclic edge colouring of middle, central, total and line graphs of prime related star graph families. Also exact values of acyclic chromatic indices of such graphs are derived and some of their structural properties are discussed.

\section{Keywords}

Acyclic Edge Colouring, Acyclic Chromatic Index, Middle Graph, Central Graph, Total Graph, Line Graph

\section{Introduction}

All graphs considered in this paper are finite, undirected and simple. The concept of acyclic colouring of a graph was introduced by B. Grunbaum [1]. A proper edge colouring of a graph $G=(V, E)$ with vertex set $V$ and edge set $E$, is a map $f: E \rightarrow C$, where $C$ is the set of colours with $f(x) \neq f(y)$ for any adjacent edges $x, y$ of $E$. The minimum number of colours needed to properly colour the edges of $G$, is called the chromatic index of $G$ and is denoted by $a^{\prime}(G)$. A proper edge colouring $f$ is called acyclic if there are no bichromatic cycles in the graph. The acyclic edge chromatic number or acyclic chromatic index, denoted by $a^{\prime}(G)$, is the minimum number of colours in an acyclicedge colouring of $G$.

Consider the set $X$ of lines of a graph $G$ with at least one line as a family of 2-point subsets of $V(G)$. The line graph [2] of graph $G$, denoted by $L(G)$, is the intersection graph $\Omega(X)$. Thus the points of $L(G)$ are the lines of $G$, with two points of $L(G)$ which are adjacent whenever the corresponding lines of $G$ are.

Let $G$ be a graph with vertex set $V(G)$ and edge set $E(G)$. The middle graph [3] of $G$, denoted by $M(G)$ is a graph with vertex set $V(G) \cup E(G)$ in which two vertices $x, y$ are adjacent in $M(G)$ if one of following 
holds. (i) $x, y$ are in $E(G)$ and $x, y$ are adjacent in $G$; (ii) $x$ is in $V(G), y$ is in $E(G)$, and $x, y$ are incident in $G$.

Let $G$ be a finite simple graph. The central graph [4] of a graph $G$, denoted by $C(G)$ is obtained by subdividing each edge of $G$ exactly once and joining all the non-adjacent vertices of $G$.

Let $G$ be a graph with vertex set $V(G)$ and edge set $E(G)$. The total graph [2] [3] of $G$, denoted by $T(G)$ is a graph with vertex set $V(G) \cup E(G)$ in which two vertices $x, y$ are adjacent in $T(G)$ if one of the following holds. (i) $x, y$ are in $V(G)$ and $x$ is adjacent to $y$ in $G$; (ii) $x, y$ are in $E(G)$ and $x, y$ are adjacent in $G$; (iii) $x$ is in $V(G), y$ is in $E(G)$, and $x, y$ are incident in $G$.

Determining $a^{\prime}(G)$ is a hard problem both from a theoretical and from an algorithmic point of view. Even for the simple and highly-structured class of complete graphs, the value of $a^{\prime}(G)$ is still not determined exactly. It has also been shown by Alon and Zaks [5] that determining whether $a^{\prime}(G)<3$ is NP-complete for an arbitrary graph $G$.

Alon, Sudakov and Zaks [6] proved that $a^{\prime}(G) \leq \Delta+2$ for almost all $\Delta$-regular graphs. This result was improved by Nesetril and Wormald [7] who showed that for a random $\Delta$-regular graph $a^{\prime}(G) \leq \Delta+1$.

In view of the discussion relating acyclic edge colouring to perfect 1-factorization conjecture, it may be inferred that finding the exact values of $a^{\prime}\left(K_{n}\right)$ for every $n$ seems hard. However, Alon et al. [8] designed an algorithm that can acyclically edge colour $K_{p}$. Through this work, they constructively showed that $a^{\prime}\left(K_{p}\right)=p$.

\section{Acyclic Edge Colouring of Line Graph of a Star Graph}

\section{Theorem}

The acyclic chromatic index, $a^{\prime}\left(L\left[K_{1, p}\right]\right)=p$, for prime $p \geq 3$.

\section{Proof}

As the line graph of the star graph is isomorphic to the complete graph and by Alon et al. [8], $a^{\prime}\left(L\left[K_{1, p}\right]\right)=p$.

\section{Acyclic Edge Colouring of Middle Graph of a Star Graph}

\section{Theorem}

For the star graph $K_{1, p-1}$ the acyclic chromatic index, $a^{\prime}\left[M\left(K_{1, p-1}\right)\right]=p$, where $p$ is prime, $p \geq 2$.

\section{Proof}

Let the edge set $E\left(K_{1, p-1}\right)=\left\{e_{1}, e_{2}, e_{3}, \cdots, e_{p-1}\right\}$ and vertex set $V\left(K_{1, p-1}\right)=\left\{v_{1}, v_{2}, v_{3}, \cdots, v_{p-1}, v_{0}\right\}$ with $v_{0}$ as the root vertex. By definition, in the middle graph $M\left(K_{1, p-1}\right)$, the vertex set is

$V\left(M\left(K_{1, p-1}\right)\right)=\left\{v_{i}: 1 \leq i \leq p-1\right\} \bigcup\left\{e_{i}: 1 \leq i \leq p-1\right\} \bigcup\left\{v_{0}\right\}$. From the definition of middle graph the vertices $\left\{e_{1}, e_{2}, e_{3}, \cdots, e_{p-1}, v_{0}\right\}$ induce a clique of order $p$, say $K_{p}$ in $M\left(K_{1, p-1}\right)$. See Figure 1 , given below. Now assign a proper colouring to the vertices of $M\left(K_{1, p-1}\right)$ as follows. Consider the colour class $C=\{0,1,2, \cdots, p-1\}$. Assign the colour $i$ to the edges $\{x, y\}$ as follows.

$$
f\left(\left\{x_{i}, y_{j}\right\}\right)= \begin{cases}2 i(\bmod p), & \text { if } i=j \\ i+j(\bmod p), & \text { if } x_{i} \text { and } y_{j} \in V\left(K_{p}\right)\end{cases}
$$

One can easily check that it is an acyclic edge colouring of $M\left(K_{1, p-1}\right)$ and hence $a^{\prime}\left(M\left(K_{1, p-1}\right)\right) \leq p$. Also $a^{\prime}\left(M\left(K_{1, p-1}\right)\right) \geq a^{\prime}\left(K_{p}\right)=p$. Hence $a^{\prime}\left(M\left(K_{1, p-1}\right)\right)=p$.

\section{Example 3.1.}

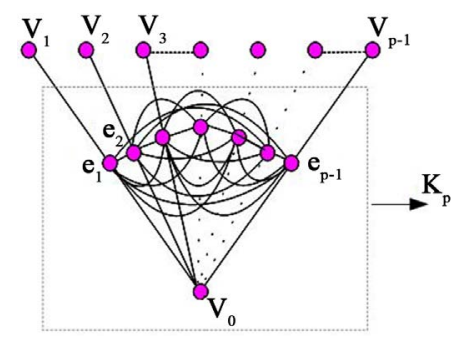

Figure 1. $M\left(K_{1, p-1}\right)$. 


\section{Acyclic Edge Colouring of Central Graph of a Star Graph}

\subsection{The Structural Properties Central Graph of Star Graph}

- The maximum degree in the graph $C\left(K_{1, n}\right)$ is $\Delta\left(C\left(K_{1, n}\right)\right)=n$.

- The minimum degree in the graph $C\left(K_{1, n}\right)$ is $\delta\left(C\left(K_{1, n}\right)\right)=2$.

- Number of edges in the graph $C\left(K_{1, n}\right), q\left(C\left(K_{1, n}\right)\right)=\left[n^{2}+3 n\right] / 2$.

- The number of vertices in $C\left(K_{1, n}\right), p\left(C\left(K_{1, n}\right)\right)=2 n+1$.

\subsection{Theorem}

For the graph $K_{1, p}$ the acyclic chromatic index $a^{\prime}\left[C\left(K_{1, p}\right)\right]=p$, for prime $p \geq 3$.

\section{Proof}

Let $V\left(K_{1, p}\right)=\left\{v_{0}, v_{1}, v_{2}, v_{3}, \cdots, v_{p-1}, v_{p}\right\}$ with $v_{p}$ as the root vertex. In central graph $C\left(K_{1, p}\right)$, by the definition each edge $v_{p} v_{i}$ for $0 \leq i \leq p-1$ of $K_{1, p}$ is subdivided by the vertex $u_{i}$ in $C\left(K_{1, p}\right)$. i.e. $V\left(C\left(K_{1, p-1}\right)\right)=\left\{v_{i}^{p}: 0 \leq i \leq p-1\right\} \bigcup\left\{u_{i}: 0 \leq i \leq p-1\right\} \bigcup\left\{v_{p}\right\}$. Now the vertices $\left\{v_{0}, v_{1}, v_{2}, v_{3}, \cdots, v_{p-1}\right\}$ induce a clique of order $p$, say $K_{p}$ in $C\left(K_{1, p}\right)$. See Figure 2 .

Now assign a proper colouring to the vertices of $C\left(K_{1, p}\right)$ as follows. Consider the colour class $C=\{0,1,2, \cdots, p-1\}$. Assign the colour $i$ to the edges $\{x, y\}$ as follows.

$$
f\left(\left\{x_{i}, y_{j}\right\}\right)= \begin{cases}i+j(\bmod p), & \text { if } x_{i} \text { and } y_{j} \in V\left(K_{p}\right) \\ 2 i(\bmod p), & \text { if } i=j \\ 2 i+2(\bmod p), & \text { if } j=p\end{cases}
$$

One can easily check that it is an acyclic edge colouring of $C\left(K_{1, p}\right)$ and hence $a^{\prime}\left(C\left(K_{1, p}\right)\right) \leq p$. Also $a^{\prime}\left(C\left(K_{1, p}\right)\right) \geq a^{\prime}\left(K_{p}\right)=p$. Hence $a^{\prime}\left(C\left(K_{1, p}\right)\right)=p, p \geq 3$.

\section{Example 4.2.}

\section{Acyclic Edge Colouring of Total Graph of a Star Graph}

\subsection{Structural Properties of $T\left(K_{1, n}\right)$}

- The maximum degree in the graph $T\left(K_{1, n}\right)$ is $\Delta\left(T\left(K_{1, n}\right)\right)=2 n$.

- The minimum degree in the graph $T\left(K_{1, n}\right)$ is $\delta\left(T\left(K_{1, n}\right)\right)=2$.

- The number of edges in the graph $T\left(K_{1, n}\right)$, is $q\left(T\left(K_{1, n}\right)\right)=\left[n^{2}+5 n\right] / 2$.

- The number of vertices in $T\left(K_{1, n}\right)$, is $p\left(T\left(K_{1, n}\right)\right)=2 n+1$.

\subsection{Theorem}

For any star graph $K_{1, p-1}$ the acyclic chromatic index, $a^{\prime}\left[T\left(K_{1, p-1}\right)\right]=2(p-1)$, for prime $p \geq 3$.

Proof

Let $E\left(K_{1, p-1}\right)=\left\{e_{1}, e_{2}, e_{3}, \cdots, e_{p-1}\right\}$ and $V\left(K_{1, p-1}\right)=\left\{v_{1}, v_{2}, v_{3}, \cdots, v_{p-1}, v_{0}\right\}$ with $v_{0}$ as the root vertex. By definition, in the total graph $T\left(K_{1, p-1}\right)$, the vertex set is $V\left(T\left(K_{1, p-1}\right)\right)=\left\{v_{i}: 1 \leq i \leq p-1\right\} \bigcup\left\{e_{i}: 1 \leq i \leq p-1\right\} \bigcup\left\{v_{0}\right\}$. Now the vertices $\left\{e_{1}, e_{2}, e_{3}, \cdots, e_{p-1}, v_{0}\right\}$ induce a clique of order $p$, say $K_{p}$ in $T\left(K_{1, p-1}\right)$. See Figure 3.

Now assign a proper colouring to the vertices of $T\left(K_{1, p-1}\right)$ as follows. Consider the colour class $C=\{0,1,2, \cdots, p-1\} \cup\{p, p+1, \cdots, 2 p-2\}=C_{1} \cup C_{2}$, say. Assign the colour $i$ to the edges $\{x, y\}$ as follows. 


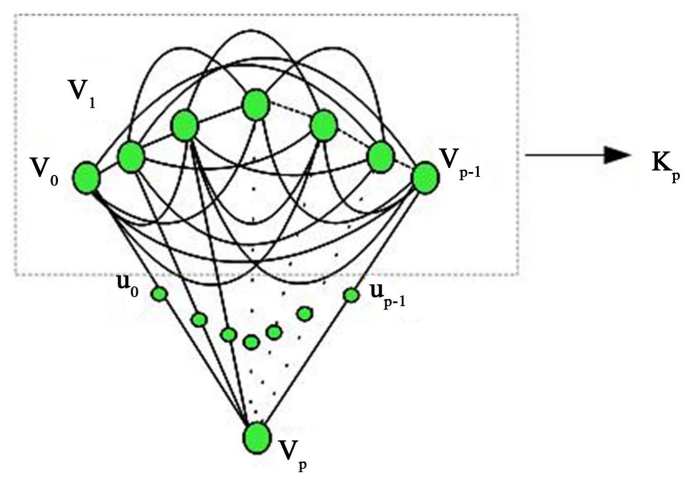

Figure 2. $C\left(K_{1, p}\right)$. Note: $a^{\prime}\left[C\left(K_{1,2}\right)\right]=3$.

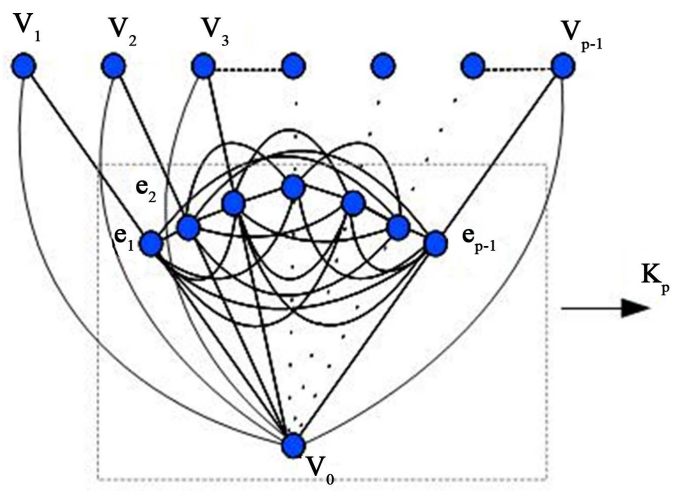

Figure 3. $T\left(K_{1, p-1}\right)$. Note: $a^{\prime}\left(T\left(K_{1,1}\right)\right)=3$.

$$
f\left(\left\{x_{i}, y_{j}\right\}\right)= \begin{cases}i+j(\bmod p), & \text { if } x_{i} \text { and } y_{j} \in V\left(K_{p}\right) \\ 2 i(\bmod p), & \text { if } i=j\end{cases}
$$

These colouring takes $p$ colours using $C_{1}$ and the remaining $p-1$ edges are assigned to the colours $C_{2}$. One can easily check that it is an acyclic edge colouring of $T\left(K_{1, p-1}\right)$ and hence $a^{\prime}\left(T\left(K_{1, p-1}\right)\right) \leq 2 p-2$. Also since $\Delta\left(T\left(K_{1, p-1}\right)\right)=2(p-1)$, minimum $2(p-1)$ colours are required for its proper edge colouring.

Hence $a^{\prime}\left(T\left(K_{1, p-1}\right)\right)=2(p-1), p \geq 3$.

\section{Example 5.2.}

\section{References}

[1] Grunbaum, B. (1973) Acyclic Colourings of Planar Graphs. Israel Journal of Mathematics, 14, 390-408. http://dx.doi.org/10.1007/BF02764716

[2] Harrary, F. (1969) Graph Theory. Narosa Publishing House, New Delhi.

[3] Michalak, D. (1983) On Middle and Total Graphs with Coarseness Number Equal 1. Lecture Notes in Mathematics, 1018, 139-150. http://dx.doi.org/10.1007/BFb0071624

[4] Thilagavathi, K. and Vernold Vivin, J. and Akbar Ali, M.M. (2009) On Harmonious Colouring of Central Graphs. Advances and Applications in Discrete Mathematics, 2, 17-33.

[5] Alon, N. and Zaks, A. (2002) Algorithmic Aspects of Acyclic Edge Colorings. Algorithmica, 32, 611-614. http://dx.doi.org/10.1007/s00453-001-0093-8

[6] Alon, N., Sudakov, B. and Zaks, A. (2001) Acyclic Edge-Colorings of Graphs. Journal of Graph Theory, 37, $157-167$. 
http://dx.doi.org/10.1002/jgt.1010

[7] Nesertril, J. and Wormald, N.C. (2005) The Acyclic Edge Chromatic Number of a Random d-Regular Graph Is d + 1 . Journal of Graph Theory, 49, 69-74. http://dx.doi.org/10.1002/jgt.20064

[8] Alon, N., McDiarmid, C. and Reed, B. (1991) Acyclic Colouring of Graphs. Random Structures \& Algorithms, 2, $277-$ 288. http://dx.doi.org/10.1002/rsa.3240020303 